64

Notre Dame Journal of Formal Logic

Volume X, Number 1, January 1969

\title{
PEIRCE'S DEVELOPMENT OF QUANTIFIERS AND OF PREDICATE LOGIC
}

\author{
RICHARD BEATTY
}

\begin{abstract}
Although there is adequate reference in the literature to the fact that Peirce did develop quantifiers, ${ }^{1}$ I know of no study of their evaluation in his thought. This topic would seem to be of sufficient historical and theoretical interest to justify its presentation here.*

Charles Peirce had a metaphysical interest in logic. Throughout his career he was concerned with the problem of the categories, and, for a good part of this career, he thought that the key to the categories was to be found in logic. This interest in the categories seems to have been the stimulus of much of his early research in logic. And it was because of this interest that Peirce was attempting to elucidate the fundamental structure of logic and to develop a notation adequate for its treatment. (cf., e.g., 3.322) It was through these repeated attempts at an adequate notation that Peirce eventually arrived at the notion of quantifiers as we know them today.
\end{abstract}

In 1867, Peirce published his first paper dealing with Boole's logic. In that same year he also published his first papers on the problem of the categories and the theory of logic upon which his deduction of the categories was based. In this paper, I shall give a very general account of the relevant features of the theory of logic of 1867 and trace the subsequent developments that eventually led, in 1885, to quantifiers, and to predicate logic.

Peirce presented his deduction of the categories in 1867 in the paper "On a New List of Categories." (1.545-559) This paper was preceded by another in the same journal, entitled "On the Natural Classification of Arguments," (2.461-516) in which he described the theory of logic his

*This paper is part of a dissertation written under the direction of Professor Otto Bird and submitted to the Graduate School of the University of Notre Dame in partial fulfillment of the requirements for the degree of Doctor of Philosophy with philosophy as major subject in August, 1966. Throughout this paper reference is made to [1] by means of bracketed numerals. For instance, " 3.322 " of the next paragraph refers to volume three, the section numbered 322 . 
deduction presupposed. This theory can be described as a subject-predicate theory insofar as Peirce held that all propositions, including conditional and relational ones, were essentially of the subject-predicate form, i.e., they each contained a subject, a copula, and a predicate. ${ }^{2}$ In the development of this theory Peirce claimed that, aside from the subject-term and copula, there were three sorts of terms that could be predicate. In a short time, the functions provided by the subject and copula seem to be subsumed under those of the three sorts of predicate, and we are left with a classification of three sorts of terms. These can be described roughly as terms representing qualities, terms representing dual relations, and terms representing triple relations. (Peirce held that all larger relational terms could be reduced to combinations of triples but that triples could not be reduced to duals.)

In 1870, Peirce published the article "Description of a Notation for the Logic of Relatives, Resulting from an Amplification of the Conceptions of Boole's Calculus of Logic." (3.45-149) His purpose in the article is to try to extend the methods of Boole's logical algebra to include not only absolute or qualitative terms, but also relative terms. De Morgan had already been working with relative terms, but with a more clumsy system of notation. Peirce was interested in this topic because it was closely related to his classification of terms and the theory of categories in general.

I have been concerned to outline the general logical context in which Peirce is working, since the development of quantifiers is closely connected with developments in the logic of relatives. I should now like to turn to the 1870 paper on a notation for relatives to consider Peirce's first attempts to express particular propositions. This is one of the main problems Peirce inherited from Boole. Before examining Peirce's first attempts to solve this problem, however, I shall give a general description of the notational methods he will be using.

As we have said, Peirce divides terms into three main types: absolute terms designating qualities, simple relative terms designating dual relations, and conjugative terms designating triple or larger relations. ${ }^{3}$ Each of these types of terms is represented by a different sort of typescript: absolute terms by letters of the Roman alphabet; simple relatives by italic letters; and conjugative terms by a sort of type called Kennerly. With all three classes of terms, small letters always denote a general term, capitals individual terms. Thus " $\mathrm{h}$ " could denote "horses"; " $\mathrm{H}$ ", " $\mathrm{H}$ " "; etc., terms designating individual horses. In regard to relative terms " $l$ ", for example, could denote "lover", and " $L$ "', etc., names of names of individual lovers.

Notice that all the terms, absolute or relative, are substantives. This is a result of the subject-predicate theory of proposition that Peirce held from 1867 until 1885, when he developed his theory of quantification (and predicate logic). In this paper of 1867, "On the Natural Classification of Arguments," Peirce developed a theory of logic in which all propositions were considered to be essentially of subject-predicate form, or, more precisely, subject-copula-predicate form. Thus, when a proposition was 
grammatically expressed in its proper logical form, even relational words would occur as predicates with a copula and thus as substantives. For example, "Boston is less than the Universe," could be translated "The relation of Boston to the Universe is a relation of less to greater." (2.507) Thus when Peirce is speaking of relatives in this early period, he is concerned with substantives rather than verbs. This is suggested even by the terminology he uses, i.e., he speaks of the logic of relatives rather than of the logic of relations.

Peirce's development of quantifiers was an answer to a problem which he had been working on for several years. The problem was how to express particular propositions. Boole's attempt to express them using an arbitrary letter representing some indeterminate class was not successful. Peirce began to wrestle with the problem in this paper of 1870 . He experimented with several different approaches. For example,

Particular propositions are expressed by the consideration that they are contradictory of universal propositions. Thus, as $h,(1-b)=0$ means every horse is black, so $0^{\mathrm{h},(1-\mathrm{b})}=0$ means that some horse is not black; and as $h, b=0$ means that no horse is black, so $0^{\mathrm{h}, \mathrm{b}}=0$ means that some horse is black. (3.141)

Zero raised to an exponent is, for Peirce, a function the value of which "vanishes" when the exponent does not, and does not "vanish" when the exponent does. (3.139)

Peirce also expresses particular propositions by using signs of inequality. Thus he writes "a, $\mathrm{h}>0$ " for "some animals are horses." (3.143) In other words, the class of things that are animals and horses is not an empty class. He suggests still another method to express such propositions. Considering a general term " $\mathrm{m}$ " to be "separated" into its individuals, $M^{\prime}, M^{\prime \prime}, M^{\prime \prime \prime}$, etc. then " $M^{\prime}$ '+ $M^{\prime \prime}$ '+ $M^{\prime \prime \prime}$ '+ etc." means "some man," and can be expressed by " $\mathrm{m}$ "'. (3.146) Thus " $\mathrm{x}-<\mathrm{y}$ ", means "some $\mathrm{x}$ is $\mathrm{y}$. " The sign " "+" represents inclusive logical addition. The sign "_<" represents Peirce's copula and can stand indifferently for "is," "if. .. then," or the illative relation, "therefore," (3.175n.1)

Considering the future development of quantification, the interesting thing about this last representation of a particular proposition is the use of a logical sum to express "some," even though the sum is taken only of the individuals of a certain class, rather than of all the individuals in the universe of discourse. The idea behind it, of course, is that to say anything is true of some of a class is to say it is true of this member, or that member, etc. Thus to say "some men are white," is to say "John is white, or Fred is white, or George, or Tom,...." In a similar manner, a logical product might be used to represent "all." Thus "all men are white" would be equivalent to "John is white, and Fred is white,...." The difficulty in using logical sums and products to represent "some" and "all" is that some universes have an infinite number of members, while the logical sum and the logical product are functions defined as operations on a finite number of terms. 
Returning to the last example above, we should note that it is inconvenient, (from the point of view concerned with the development of a facile notation), to represent "some" of a class by a logical sum of its members e.g., " $\mathrm{h}=\mathrm{H}^{\prime}$ ' $+\mathrm{H}^{\prime \prime}$ ' $+\mathrm{H}^{\prime \prime}$ " $+\ldots .$. ', This can be seen clearly enough in comparison with the currently received methods of predicate logic. Suppose, for example, you are given two propositions, "Some horses are thorobreds," and "Some black things are four-legged," and you want to express, further, that the horses and black things referred to are identical. With Peirce's notation of this period, you would have two sets of individual

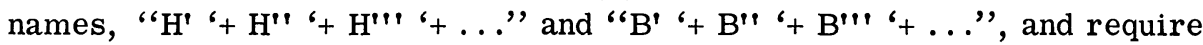
some method of showing, at least theoretically, that particular names named the same objects. This, of course, would be impractical to the point of impossibility. If, as in current methods, you had variables which ranged over all the individuals, having a purely referential function and no connotation, you could easily express that the same individuals had the characteristics in question. The current notation is superior to that above in that it involves the use of variables and the separation of the connotative and denotative or referential functions.

We should note that there is an ambiguity in Peirce's method of notation here since Peirce represents all general terms as logical sums of the individual terms of the same class. Thus,

$$
\begin{aligned}
& \mathrm{h}=\mathrm{H}^{\prime}+\mathrm{H}^{\prime}{ }^{\prime}+\mathrm{H}^{\prime \prime} \text { ' }+ \text { etc. } \\
& l=L^{\prime}+L^{\prime}{ }^{\prime}+L^{\prime \prime}{ }^{\prime}+\text { etc. }
\end{aligned}
$$

Thus, " $\mathrm{H}$ ' $+\mathrm{H}^{\prime}$ ' $+\mathrm{H}^{\prime \prime}$ '+ etc." could be interpreted either as the analysis of the general term "horse" or as the quantified expression "some horse."

When Peirce finally arrived at the notion of quantifiers, he used the letter " $\Sigma$ " to represent the particular quantifier. " This letter was used in his second paper on Boole's algebra (1867, 3.20-44) to represent a logical sum, (3.21) and was used in this 1870 paper, (again representing the taking of a sum), to represent "some" of a class. It occurs, for example, in the expression

$$
e^{f}+, \Sigma_{p} e^{f-p}, c^{p}+, c^{f}
$$

which denotes "whatever is emperor of every Frenchman or emperor of some Frenchmen and conqueror of all the rest, or conqueror of every Frenchman." (3.77) The comma stands for logical multiplication, (3.53, $3.73-74)$ the plus sign with a comma stands for inclusive logical addition, (3.67) and the use of a letter as an exponent has the effect of universally quantifying that letter. (3.77) Thus " $l$ "' means "lover of every woman." If we separate the phrase " $\Sigma_{p} e^{f-p}, c$ " from the rest of the expression, we find its interpretation to be "emperor of some Frenchmen and conqueror of all the rest." In other words, "emperor of all Frenchmen not p's, and conqueror of all those who are p's." Given the assumption that the p's must fall under the f's, " $\Sigma$ " seems to act very much like a particular quantifier. It seems to designate indefinitely a sum of Frenchmen for removal from the scope of " $e$ " and placement in the scope of " $c$ ". 
If we turn now to a paper published in 1880 entitled "On the Algebra of Logic," (3.154-251) we find Peirce expressing propositions in the forms " $A-\angle B$ " and " $A=B$," the second being the negative or contradictory of the first. (3.175) This second proposition, however, can be expressed as " $\breve{A}-<\bar{B}$," where " $\breve{A}$ " means "some $A$ " and " $\bar{B}$ " means "not- $B$." Apparently the form " $A-\angle B$ " is to represent "All $A$ is $B$," and the form " $A=B$ " "It is not the case that all $A$ is $B$." The four traditional propositions can now be expressed with this terminology, but with no existential import for universal propositions. (3.176-178)

As we have already mentioned, in 1870 Peirce considered a general term to be equivalent to the logical sum of the names of the individuals contained in the class designated by the general term. In 1880 , he seems to remove this restriction of the individuals named to the class involved. "Every term may be conceived as a limitless logical sum of individuals." (3.217) Since he says every term may be so conceived, this would apply to, among others, individual terms and terms designating classes with a finite number of members. And since neither of these sorts of terms designate classes containing a limitless number of individuals, Peirce seems to be suggesting that any term can be considered equivalent to the logical sum of all the members of the universe of discourse. ${ }^{5}$ This equivalence will always hold, but, when terms are represented in this manner, you have no way of distinguishing one term from any other; for they are all equivalent to the same limitless logical sum. Peirce deals with this problem in 1882, (as we shall see below), by introducing coefficients modifying each term in a particular sum. The function of the coefficient is to cancel or leave intact the term it modifies. And, as a matter of fact, it is in connection with these expressions of the coefficients as they eventually become reinterpreted as expressions in predicate logic that Peirce develops his quantifiers.

Let me say more about this notion of giving the analysis of a term as a limitless logical sum of all the members of the universe of discourse. Quantification uses variables ranging over all the individuals in the universe of discourse, whether their number be finite or not. It would seem that this notion of the analysis of a term is a step toward the development of quantifiers in that it suggests the need for neutral variables of the sort used in predicate logic, i.e., variables which are purely referential and not connotative, i.e., variables which can range neutrally over all the members of the universe. This is an advance toward the conception of predicate logic, in which there is a separation of the connotative and referential functions, and which is the kind of logic in connection with which Peirce developed his quantifiers.

In accordance with his notion of the analysis of terms as sums of individuals (or, in the case of relative terms, as sums of individual relatives,) we find Peirce in this same paper representing the analysis of terms by means of sums using subscripts which are applied to the " $\Sigma$ " (or " $\Pi$ ") and the individual terms of the individual relative (or simply individual term, if such be the case,) involved. Thus, 


$$
l=\Sigma_{i}\left(\mathrm{~L}_{i}: \mathrm{M}_{i}\right)
$$

By this symbolism Peirce apparently means that the relative " $l$ " is a sum of pairs of individuals, each individual identified for its pair by the subscript or index " $i$ ". The letters " $\mathrm{L}$ " and " $\mathrm{M}$ " stand for the individuals which might be related in this manner; that the individuals are (or may be) different is indicated, of course, by the different letters, " $\mathrm{"}$ " and " $\mathrm{M}$ ". Peirce is concerned here with representing a general term in accordance with his theory of analysis. Its importance to quantification lies in the use of subscripts to both the symbols designating logical sums or products and those designating the individuals in the universe of discourse, the purpose of the subscripts being to range over these individuals indicating pairs of them (etc.) as members of this logical sum (or product, etc.). These subscripts look very much like the bound variables of quantification theory, but they are not the same. The letters referring to the individuals in the universe of discourse in the above example are not the subscripts, but rather " $\mathrm{L}$ " and " $\mathrm{M}$ ". The function of the subscripts is to differentiate a variety of pairs of "L"'s and "M"'s to be added in the sum.

As we said above, considering terms as equivalent to limitless logical sums of individual terms or individual relatives, Peirce had the problem of differentiating the analysis of one term from that of another since, for example, any two absolute terms would be equivalent to the same limitless logical sum of individuals, i.e., the sum of all the members of the universe of discourse. In 1882, Peirce dealt with this problem by introducing a numerical coefficient to each member of the sum. Any absolute term, for example, could be expressed as the sum of all the letters denoting objects, each letter modified by a coefficient. Thus,

$$
x=(x)_{a} A+(x)_{b} B+(x)_{c} C+\text { etc. }=\Sigma_{i}(x)_{i} I
$$

Apparently the function of the subscripts to the " $\Sigma$ ", and coefficients is to identify the coefficient proper to each member of the sum. Thus, the coefficient with subscript " $a$ " modifies the letter " $A$ ". The designation of the coefficient itself by the same letter as the term being analysed is to show that we are taking the coefficient proper to that term. Thus the coefficient " $(x)_{i}$ " has two references: one to the term being analysed, the other to the particular member of the sum which it modifies. In 1883, Peirce identifies these coefficients as 0 and 1, apparently their purpose is to either nullify a particular value or leave it unchanged. (3.329) Thus, Peirce can now represent any absolute term as a sum of all the individual terms in the universe of discourse, each term modified by a coefficient whose effect is to make the term belong or not belong to the sum-class. For if a term is modified by 0 , the resultant value of the combination is 0 , and 0 adds nothing to a sum. If a term is modified by 1 the value of the combination is the same as that of the term itself, and the term will be a member of the sum-class. Thus we can explain the equation " $x=\Sigma_{i}(x)_{i} I$ ", in the following way: The first " $x$ " stands for the term to be expressed as a sum of individuals. The " $I$ " stands for all the individual terms in the universe of discourse. The " $x$ " enclosed in parentheses is a coefficient 
modifying " $I$ ". The " $\Sigma$ " signifies that a sum of all the " $I$ "'s modified by coefficients is to be taken. The subscript to the " $\Sigma$ " and to the coefficient signifies which value of the coefficient is to be taken with each "I" in the sum. The representation of the coefficient as well as the term to be expressed by " $x$ " suggests that one pick out the correct coefficient for a particular individual by knowing whether or not the individual is properly named by the term " $x$ ". In this last respect, this method of expressing terms does not seem any more advanced than Peirce's earlier attempts to represent general terms by the sum of the individuals falling under them; e.g., " $h=H$ ' $+H^{\prime}$ '+ $H^{\prime \prime}$ "+ etc." There seems to be an advance in this respect, however, that Peirce is moving away from a system of expression in which terms denoting individuals have connotation and moving toward a terminology which uses individual variables having no connotation and applying to all the individuals in the universe of discourse. The capital "I" in the above example is a genuine individual variable. Even the representation of the individual names seems to show this change since they are now given as $A, B, C$, etc. rather than, as in the earlier example concerning "horse," $\mathrm{H}, \mathrm{H}$ ', $\mathrm{H}$ " etc.

A similar notation also applies to relatives. Thus "If $l$ denotes any general dual relative, then the coefficient of the pair $I: J$ in $l$ is written $(l)_{i \breve{j} .} . "(3.309)$ For example, Peirce represents the self-relation as follows:

$$
\mathrm{S} x=\Sigma_{i}(x)_{i i}(I: I)
$$

and external multiplication, (relative product), as

$$
(x y)_{i j}=\Sigma_{n}(x)_{i n}(y)_{n j}
$$

Notice in the latter case that Peirce has omitted the expressions such as " $(I: J)$ " which refer to the individual relative, since everything they express is expressed equally well by the subscripts to the coefficients alone. The role of the subscripts has been to refer the correct coefficient to the appropriate individual relatives. Without the expressions for the individual relatives, the subscripts can be taken as referring to the individuals themselves. But, as we shall see, the omission is only for the sake of brevity; the expression, e.g., " $(x)_{i n}$ " is still to be understood as a coefficient modifying an individual relative.

In an article of 1883 entitled "The Logic of Relatives," (3.328-358) Peirce once again presents his analysis of relative terms in the following manner,

$$
l=\Sigma_{i} \Sigma_{j}(l)_{i j}(I: J)
$$

but again the appendage " $(I: J)$ " representing the individuals in the universe of discourse is soon omitted, and terms are expressed in terms of sums and products of numerical coefficients alone.

Peirce also omits the same element from his expression of propositions:

Any proposition whatever is equivalent to saying that some complexus of aggregates and products of such numerical coefficients is greater than zero. Thus, 


$$
\Sigma_{i} \Sigma_{j} l_{i j}>0
$$

means that something is a lover of something;

$$
\Pi_{i} \Sigma_{j} l_{i j}>0
$$

means that everything is a lover of something. We shall, however, naturally omit, in writing the inequalities, the $>0$ which terminates them all; and the above two propositions will appear as $\Sigma_{i} \Sigma_{j} l_{i j}$ and $\Pi_{i} \Sigma_{j} l_{i j}$. (3.351)

This is a very significant development. Peirce is only a step away from the notion of predicate logic and quantification.

First, Peirce says that any proposition can be expressed as a complexus of sums and products of coefficients greater than zero. He then translates the first formula given in the quotation as "something is a lover of something." The explanation of how that formula can be said to express that proposition is the following: "I" and " $J$ " represent all the individuals in the universe of discourse, only we use two different letters since they do not necessarily represent the same individual at the same time. If there is no pair of individuals, $I$ and $J$, such $I$ loves $J$, then the coefficients of all the pairs, $I: J$ are always zero, and the sum of these coefficients is also zero. If there is at least one pair, $I: J$, such that $I$ loves $J$, then the coefficient of this pair is one. And a sum of coefficients at least one of which has the value "one" will always be equivalent to one, i.e., it will be greater than zero. Thus, if the complexus of sums " $\Sigma_{i} \Sigma_{j} l_{i j}$ " is greater than zero, there must be something which loves something.

Notice that although the expression " $l_{i j}$ " stands for a coefficient, it also expresses the relation $(l)$ and individuals $(i, j)$ which the coefficient modifies. It would be easy to interpret the expressions, not as coefficients involving reference to the related pairs of which they are coefficients, but as propositions expressing relations between individuals and having the value 1 when individuals are so related and 0 when they are not so related. That is, the expression " $l_{i j}$ " may be interpreted in the usual way as expressing a proposition having a value, rather than as a coefficient referring to the relation of which it is coefficient.

Lastly, Peirce says that we can omit the expression ">0" when we write out formulas. The idea is simply that we establish the convention that if any proposition is written down it shall be understood as having a value greater than zero--unless otherwise indicated, of course. ${ }^{6}$

In the paper of 1885 , we find the first explicit use of the term "quantifying" (3.393) and the actual development of quantification itself. Peirce remarks on the past failure to introduce the terms "some" and "all" into Boolean algebra and attributes the final success in this regard to $\mathrm{O} . \mathrm{H}$. Mitchell. ${ }^{7}$ Mitchell, he says, divided propositions into two parts, "a pure Boolean expression referring to an individual and a Quantifying part saying what individual this is." (3.393) Thus, using the symbol " $U$ " to stand for the universe of class terms, (which may be limited or unlimited), ${ }^{8}$ Mitchell represents the proposition "All $U$ is $F$ " by " $F_{1}$ " and the proposition "Some $U$ is $F$ " by " $F$ ". . The subscript " 1 ", signifies that the class term " $F$ " is true of all members of the universe of class terms, and the subscript " $u$ " 
signifies that it is true of some members. With this notation Mitchell can now represent the four Aristotelian propositions in the following way:

$\begin{array}{ll}\text { No } a \text { is } b . & =(\bar{a}+\bar{b})_{1} \\ \text { Some } a \text { is } b . & =(a b)_{u} \\ \text { All } a \text { is } b . & =(\bar{a}+b)_{1} 10 \\ \text { Some } a \text { is not } b . & =(a \bar{b})_{u}\end{array}$

You can interpret the symbolism of the first proposition as follows: All of $U$ is $\overline{\mathrm{a}}+\overline{\mathrm{b}}$, i.e., nothing is both $a$ and $b$, or no $a$ is $b$. The second: Some of $U$ is ab, i.e., something is both $a$ and $b$, i.e., some $a$ is $b$. And so on.

It is clear then that Mitchell has introduced a sort of quantification which can be used to express universal or particular propositions even in an unlimited universe of discourse. But there is still room for improvement in this notation. As Mitchell himself says, "When $u$ is written as a suffix of different propositions in the same argument, it is not meant that the same part of $U$ is concerned in each case."11 For example, (using " + " for both class and propositional disjunction as Mitchell does), we can interpret the expression

$$
(\mathrm{a})_{u}+(\mathrm{b})_{u}
$$

as we would

$$
\Sigma \mathrm{xFx}+\Sigma \mathrm{xGx}
$$

that is, as not necessarily referring to the same part of the universe as being either $a$ or $b$, or as being $F$ or $G$. A reference to the same part of the universe would require expressions with a single quantifier, as

$$
(\mathrm{a}+\mathrm{b})_{u} \text { or } \Sigma \mathrm{x}(\mathrm{Fx}+\mathrm{Gx}) \text {. }
$$

The difficulty would occur when one is required to make multiple references to the same part of the universe of discourse, as in the expression, "everyone loves someone who benefits everyone." Extending Mitchell's notation to apply to relatives, the closest we could come would be " $1_{1 u} b_{u 1}$ ". This says, "everyone loves someone and someone benefits everyone," but not necessarily the same "someone." The standard quantificational methods, however, (i.e., Peirce's methods), can express it as follows: " $\Pi x \Sigma y 1 x y b y x "$. The separation of the quantifying and indexing functions in different symbols allows Peirce to use the same index multiple times with the same quantifier.

Thus, in 1885, undoubtedly stimulated by Mitchell's work, Peirce developed quantifiers as we know them today.

We may use $\Sigma$ for some, suggesting a sum, and $\Pi$ for all, suggesting a product. Thus $\Sigma_{i} x_{i}$ means that $x$ is true of some one of the individuals denoted by $i$ or

$$
\Sigma_{i} x_{i}=x_{i}+x_{j}+x_{k}+\text { etc. }
$$

In the same way, $\Pi_{i} x_{i}$ means that $x$ is true of all these individuals, or

$$
\Pi_{i} x_{i}=x_{i} x_{j} x_{k} \text {, etc. }
$$


If $x$ is a simple relation, $\Pi_{i} \Pi_{j} x_{i j}$ means that every $i$ is in this relation to every j. . . (3.393)

But, "it is to be remarked that $\Sigma_{i} x_{i}$ and $\Pi_{i} x_{i}$ are only similar to a sum and a product; they are not strictly of that nature, because the individuals of the universe may be innumerable." (3.393)

Thus we have finally reached the notion of quantifiers. The " $\Sigma$ " and " $\Pi$ " only suggest a sum and product; they may operate upon an infinite number of individuals but a sum and product may not. Notice also that in the expression " $x_{i j}$ " the " $x$ " stands for a relation and the indices refer to individuals. We have seen above that the same sort of expression was used to express coefficients.

Let me summarize the developments that led to quantifiers.

Peirce began, in 1867, with a subject-predicate theory of logic, a theory he held to until 1885, the year he developed quantifiers and predicate logic. This theory involved a classification of all terms into three sorts: absolute terms, dual relatives, and triple relatives. These terms appeared in propositions as subjects and predicates in the traditional sense and thus were substantives (or substantival phrases).

Peirce inherited the problem of expressing particular propositions from Boole. In 1870, he tried various methods of solving this problem. One method that was relevant for his later development was the representation of "some" of a class by the logical sum of all the members of that class.

One ambiguity of Peirce's thought at this time is that he also presents the analysis of a general term as a logical sum of the individual terms representing the members of the class designated by that term. In 1880 , he presents terms as being analysable in terms of a limitless logical sum of all the individual terms in the universe of discourse (or, in the case of relatives, of all the pairs of individual terms). As I suggested above, this seems to be an advance toward the notion of quantifiers in that it suggests the need for neutral variables ranging over all the members of the universe of discourse and as a consequence, there is a suggestion of a logic separating the connotative and referential functions, i.e., a predicate logic of the sort for which Peirce developed quantifiers.

Another notational method that Peirce used in 1880 that was to be important for the development of quantifiers was the use of subscripts in the representation of the analysis of terms, e.g., " $l=\Sigma_{i}\left(\mathrm{~L}_{i}: \mathrm{M}_{i}\right)$ "'. The letters " $\mathrm{L}$ " and " $\mathrm{M}$ " are individual variables ranging over the universe of discourse; the function of the subscripts is to pick out pairs of the individual terms, all of which, of course, are members of the logical sum representing the analysis of the term $l$. These subscripts, when applied to coefficients, will eventually evolve into the variables bound by the quantifiers.

In 1882, Peirce once again represents the analysis of a term by a limitless logical sum of individual terms (or pairs of terms, etc.), but now he introduces coefficients modifying each member of the sum, their effect being either to cancel the term modified or leave it intact. E.g., 
" $x=\Sigma_{i}(x)_{i} I$ " Peirce frequently omits the individual variables like " $I$ " since the subscripts to the coefficient refer to the variable involved. This possibility of omitting part of the expression suggests, of course, that part is superfluous. But that part is not really superfluous until the whole expression is given a new interpretation. This Peirce eventually did under the influence of $\mathrm{O}$. $\mathrm{H}$. Mitchell.

In 1883, Peirce expresses propositions in terms of the coefficients and the sign " $>0$ ". E.g., " $\Sigma_{i} \Sigma_{j} l_{i j}>0$ " means "something is a lover of something." The idea is that if a complexus of sums and products of coefficients is "greater than zero," then the complex term of which the coefficients are a part is satisfied.

I should point out here again that expressions like " $\Sigma_{i} \Sigma_{j} l_{i j}$ " are still not to be interpreted as expressing propositions directly, but as complexes of coefficients. Every proposition that Peirce has expressed in symbolic notation up until and including this paper has involved his sign for the copula or a symbol definable in terms of the copula. At this time he is still working in the context of the subject-predicate theory of logic. His expressions sometimes have the exact form of current predicate logic, but they are not to be mistaken as the same. Thus Peirce says the expression ">0" can be dropped from the above complexes of coefficients, leaving, e.g., " $\Sigma_{i} \Sigma_{j} l_{i j}$ ". But this expression still refers to a complexus of coefficients and the sign " $>0$ " is still understood to be in effect. Of course, the "natural" way to interpret such an expression is as expressing a proposition directly. This Peirce himself will do shortly, under the influence of Mitchell.

In this same year, (1883), O. H. Mitchell successfully developed a notation involving quantifiers. Mitchell represented that a class term was true of everything in the universe of discourse by using the subscript " 1 " to the class terms, and that a class term was true of some part of the universe by subscripting " $u$ " to the class term. This is a very neat notation, involving the separation of the connotative and referential functions, but in joining the referential and quantifying functions the possibility of having the same particular index subjoined to different class terms is lost. Nevertheless, Mitchell was successful in introducing into logic the expression of "all" or "some" of the universe of discourse, whether that universe be finite or infinite.

In 1885, clearly under the influence of Mitchell, Peirce developed his own set of quantifiers. After Mitchell's simple notation, it would be difficult for Peirce not to reinterpret the coefficient expressions as directly expressing propositions. Like Mitchell's notation, the referential function is accomplished by the subscript and the connotative by the letter to which the subscript is applied. But Peirce's notation improves on Mitchell's in that there is the separation of the quantifying and referring functions in different symbols. This was a natural development for Peirce since he had already been using complex coefficient functions involving logical sums and products operating by means of indices. Thus it was only a short step for Peirce to reinterpret his " $\Sigma$ " and " $\Pi$ " as quantifiers applying to a finite 
or infinite number of objects, rather than symbols for sums and products. Thus there was a two-part development: reinterpretation of the coefficient expressions as propositional expressions involving separate referential and connotative elements, and reinterpretation of the symbols for logical sum and product as applying to a possibly infinite universe.

As I said, Mitchell's notation, in conjoining the referring and quantifying functions in a single functor, does not allow one to refer specifically to the same part of the universe in separate references. For example, even if we extend Mitchell's notation in a natural way to apply to relatives, it cannot express "everyone loves someone who benefits everyone." In Mitchell's notation the closest we could come would be " $l_{1 u} b_{u 1}$ ". This says, "everyone loves someone and someone benefits everyone"--but not necessarily the same "someone." Peirce, however, can express it as follows: " $\Pi_{x} \Sigma_{y} l_{x y} b_{y x}$ ". The separation of the quantifying and indexing functions allows Peirce to use the same index more than once, all its uses being bound by a single, separate quantifier.

As we noted above, Peirce's development of quantifiers was simultaneous with his development of predicate logic. Peirce reinterpreted his coefficient expressions as quantified expressions of predicate logic. Since the subscript is now taken as a variable ranging over the members of the universe of discourse, the coefficient symbol is naturally reinterpreted as a predicate or relation. Since there is no intervening copula function, it is a verbal predicate or relation, not a substantival one.

The beauty of the methods of predicate logic as Peirce developed them seems to lie in the fact that he developed a simple notation which allowed a great richness of expression. One of the reasons for this accomplishment seems to be the separation of the three major logical functions of predication, reference, and quantification in three separate types of functors. As we have seen, the separation of the predicative and referential functions allowed a great simplification of expression, and the separation of the quantifying function from the functor referring to what's quantified allows a great enrichment of expression.

There was the further simplification of the absorption of the copulative function into the predicate, i.e., the change from substantival to verbal predicates. The copula might be desirable in some instances, e.g., if one were interested in a topic like traditional conversion. But if one is not interested in such a topic, the copula is superfluous, and, since it always appears with a predicate, it can have its function absorbed into that of the predicate.

\section{NOTES}

1. Cf. I. M. Bochenski, A History of Formal Logic, pp. 347-49; C. I. Lewis, $A$ Survey of Symbolic Logic, pp. 92-100; W. Kneale and M. Kneale, The Development of Symbolic Logic, pp. 430-32.

2. But Peirce's understanding of the subject-predicate form is peculiar in that grammatical structure is not equivalent to logical structure. For example, a 
grammatical subject might be functioning as a logical predicate. This theory of subject-predicate form is connected with his theory of thought by sign-translation.

3. Since Peirce considers the essential form of all propositions to be subjectcopula-predicate, (cf. next paragraph), it might be less misleading to speak of terms as designating objects in relation rather than as designating relations. For a relational verb like, e.g., "loves," will always occur, (in a proposition in fundamental form), as part of a substantival phrase, such as, "the one whom John loves." So when I say "term designating a relation," I have in mind a term like "lover" rather than one like "loves."

4. He used " $\Pi$ " to represent the universal quantifier. Before that it represented the taking of a logical product.

5. Of course relative terms will be equivalent to such sums of individual relatives.

6. Peirce suggests the possibility of other sums and products. "Thus, we may use $\Pi^{\prime}, \Pi^{\prime \prime}$, etc., to mean the products for all individuals except one, except two, etc." (3.357)

7. Kneale mistakenly remarks that Mitchell did not develop quantifiers as Peirce claimed because, although Mitchell used " $\Sigma$ " and " $\Pi$ " in connection with relational signs and indices, his use of such notation, unlike Peirce's, did not amount to quantified statements. Kneale errs by supposing that Mitchell's quantifiers would involve the same symbols as Peirce's, which they did not. Bochenski correctly identifies Mitchell's quantifiers. Cf. Kneale, op. cit., p. 431; Bocheński, op. cit., p. 348.

8. O. H. Mitchell, "On a New Algebra of Logic," Studies in Logic by Members of the John Hopkins University, C. S. Peirce, ed., p. 73.

9. Ibid., p. 74 .

10. Ibid., p. 75 .

11. Ibid., p. 77.

\section{REFERENCES}

[1] Collected Papers of Charles Sanders Peirce. 8 vols., vols. 1-6 edited by C. Hartshorne and P. Weiss, Cambridge, Mass., The Belknap Press of Harvard University Press, 1960; vols. 7-8 edited by A. W. Burks, Cambridge, Mass., Harvard University Press, 1958.

[2] Peirce, C. S., (ed.), Studies in Logic, Boston, Little, Brown, and Co., 1883.

[3] Bocheński, I. M., A History of Formal Logic, (Trans. and ed. by I. Thomas, Notre Dame, Indiana), University of Notre Dame Press, 1961.

[4] Kneale, W., and Kneale, M., The Development of Logic, Oxford, The Clarendon Press, 1962.

[5] Lewis, C. I., A Survey of Symbolic Logic, New York, Dover Publications, Inc., 1960. 